\title{
Fabrication of ZnO Nanowires Arrays by Anodization and High-Vacuum Die Casting Technique, and Their Piezoelectric Properties
}

\author{
Chin-Guo Kuo ${ }^{1}$, Ho Chang ${ }^{2, *}$ and Jian-Hao Wang ${ }^{2}$ \\ 1 Department of Industrial Education, National Taiwan Normal University, Taipei 10610, Taiwan; \\ chinguo7@yahoo.com.tw \\ 2 Graduate Institute of Manufacturing Technology, National Taipei University of Technology, Taipei 10608, \\ Taiwan; t101408074@ntut.edu.tw \\ * Correspondence: f10381@ntut.edu.tw; Tel.: +886-2-27712171; Fax: +886-2-27317191
}

Academic Editor: Teen-Hang Meen

Received: 19 January 2016; Accepted: 22 March 2016; Published: 24 March 2016

\begin{abstract}
In this investigation, anodic aluminum oxide (AAO) with arrayed and regularly arranged nanopores is used as a template in the high-vacuum die casting of molten zinc metal $(\mathrm{Zn})$ into the nanopores. The proposed technique yields arrayed $\mathrm{Zn}$ nanowires with an aspect ratio of over 600 . After annealing, arrayed zinc oxide $(\mathrm{ZnO})$ nanowires are obtained. Varying the anodizing time yields AAO templates with thicknesses of approximately $50 \mu \mathrm{m}, 60 \mu \mathrm{m}$, and $70 \mu \mathrm{m}$ that can be used in the fabrication of nanowires of three lengths with high aspect ratios. Experimental results reveal that a longer nanowire generates a greater measured piezoelectric current. The $\mathrm{ZnO}$ nanowires that are fabricated using an alumina template are anodized for $7 \mathrm{~h}$ and produce higher piezoelectric current of up to $69 \mathrm{pA}$.
\end{abstract}

Keywords: $\mathrm{ZnO}$ nanowires; anodic aluminum oxide (AAO); vacuum die casting

\section{Introduction}

Zinc oxide $(\mathrm{ZnO})$ is an n-type II-VI semiconductor group material with a wurtzite structure in a hexagonal crystal system. Since it is symmetric and has no center of symmetry, this structure has favorable piezoelectric properties [1]. As components are miniaturized, piezoelectric materials have become nanosized. In recent years, $\mathrm{ZnO}$ nanowires (NWs) have been used in nanogeneration devices [2,3]. In relevant works, the most representative device of this kind is the piezoelectric nanogenerator that was developed by a research team that was led by Wang [4]. First, an atomic force microscope (AFM) was used as a probe to apply stress to $\mathrm{ZnO}$ nanowires to make them produce strain, and then the piezoelectric current was measured. This property was further employed to develop a nanogenerator. Xue studied the response of a nanogenerator (NG) to the gas environment in which it is placed. Xue's experimental results indicated that the output of a piezoelectric nanogenerator (NG) that was fabricated using $\mathrm{ZnO}$ nanowire arrays is largely influenced by the density of the surface charge carriers at the nanowire surfaces [5]. Lin developed Pd nanoparticles that were uniformly loaded on the whole surfaces of $\mathrm{ZnO}$ nanowire arrays using a simple hydrothermal method. The piezoelectric output that was generated by the $\mathrm{Pd} / \mathrm{ZnO}$ nanoarray nanogenerator acted not only as a power source but also a response signal to ethanol at room temperature [6]. Fu fabricated a $\mathrm{ZnSnO}_{3} / \mathrm{ZnO}$ nanowire piezo-nanogenerator as a self-powered gas sensor with high sensitivity, selectivity, and reliability to detect liquefied petroleum gas at room temperature. The results indicated that the sensitivity of the self-powered gas sensor to 8000 ppm liquefied petroleum gas was up to 83.23, and the limit of detection was 600 ppm [7]. Zeng utilized a low-temperature hydrothermal method to fabricate vertically aligned 
$\mathrm{ZnO}$ nanowires that were grown on graphene oxide (GO) films. The results indicated that the graphene oxide layer facilitated the vertical growth of $\mathrm{ZnO}$ NWs and improved their crystal quality [8]. Lin used a solution-free, catalyst-free, vapor-phase growth method to synthesize $\mathrm{ZnO}$ nanorod arrays on Al-doped $\mathrm{ZnO}$ (AZO) films. The experimental results of Lin revealed that the electrical performance of the transparent conductive oxide films was not degraded by the growth of nanorod arrays, and the nanorods were highly crystalline [9]. Tian performed pure Zn chemical vapor deposition on an anodic aluminum oxide substrate at $950{ }^{\circ} \mathrm{C}$ to form develop multilayer comb-like zinc oxide nanostructure. The experimental results of Tian reveal that the growth of the $\mathrm{ZnO}$ nanostructures is controlled by the vapor-solid (VS) growth mechanism and is a diffusion-limited process [10].

To fabricate a one-dimensional nanostructure, Zhang et al. successfully placed bismuth (Bi) in a vacuum chamber for heating and melting, and cast the molten Bi into the alumina template using high-pressure gas [11,12]. A high temperature was used to melt the metal. The molten metal material was cast into the nanotemplate by pressurization to fabricate arrayed nanowires. In the fabrication of the nanowires, the control variables were pressure value, heating temperature, the properties of the liquid metal, and the applied force. However, the pressure of the high-pressure gas was limited by a gas compressor. To solve the problems that were caused by gas injection, mechanically powered hydraulic equipment was used to apply additional pressure in a newly developed high-vacuum die casting technique that combined traditional casting with a nano-technique [13].

Anodization was used to fabricate porous anodic aluminum oxide (AAO) templates. The pore size of the template was controlled using various solutions. Accordingly, highly regularly arrayed nanopores were formed [14]. The depth of the pores was determined by the anodizing duration. Then high-vacuum die casting technique was used to cast the material into the nanopores of the template, and thus to fabricate nanowires of different lengths. The purpose of this work is to fabricate $\mathrm{ZnO}$ nanowire with a diameter of $80 \mathrm{~nm}$ inside the nanopores of an AAO template and to study the relationship between the length of the $\mathrm{ZnO}$ nanowire and the piezoelectric properties of $\mathrm{ZnO}$ material with a one-dimensional nanostructure. The results of this research may improve our understanding of $\mathrm{ZnO}$ material on the nanoscale.

\section{Experimental Details}

In the experiment herein, porous AAO film was used as a template, on which zinc foil was placed. The template was heated until molten. Normal pressure was applied to cast the molten zinc into the nanopores of the template. Atmospheric annealing was performed to transform zinc nanowires into $\mathrm{ZnO}$ nanowires. Finally, part of the AAO nanotemplate was removed to expose the nanowires. Figure 1 shows a flow chart of the experiment.

(a) $\mathrm{AAO}$

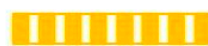

(c) $\mathrm{Etch} \mathrm{Al}_{2} \mathrm{O}_{3}$

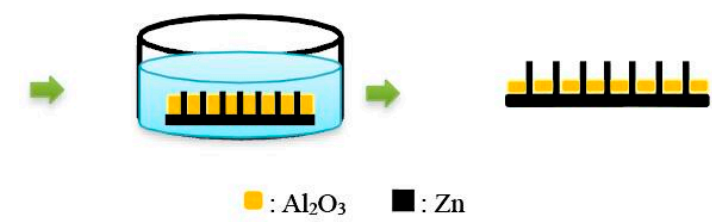

(b) Die-casting

\section{TाПता}

(d) Drying

Figure 1. Experimental process. AAO: anodic aluminum oxide.

In 1995, Masuda et al. discovered the self-assembling porous alumina film, and developed hexagonally arrayed porous alumina [15]. They used a two-step anodization method to grow alumina film. Aluminum foil with a purity of $99.7 \%$ was used as the substrate. $\mathrm{HClO}_{4}, \mathrm{C}_{2} \mathrm{H}_{5} \mathrm{OH}$, and 
$\mathrm{HOCH}_{2} \mathrm{CH}_{2} \mathrm{OC}_{4} \mathrm{H}_{9}$ were mixed uniformly in a ratio of 3:14:3 to form an electrolytic polishing solution. With pure aluminum foil as the anode and graphite foil as the cathode, electrolytic polishing of the foil was performed, completely flattening, lustering, and smoothing its surface, favoring the fabrication of the AAO film with very regularly arrayed nanopores. Thereafter, $0.3 \mathrm{M}$ oxalic acid solution was used in two-phase anodization. With aluminum foil as the anode and graphite foil as the cathode, $40 \mathrm{~V}$ voltage was applied for $1 \mathrm{~h}$ to perform phase- 1 anodization. Then, $6 \%$ phosphoric acid solution was added to $2 \%$ chromic acid solution, and the resulting solution was used to remove the AAO film that was grown in phase- 1 at $60{ }^{\circ} \mathrm{C}$. Finally, phase- 2 anodization was performed. A second anodization for 5,6, and $7 \mathrm{~h}$ yielded films of various thicknesses. As presented in Figure 2, the nanopores in the obtained AAO template were very regularly arrayed with a complete structure, and all pores had a size of $80 \mathrm{~nm}$.

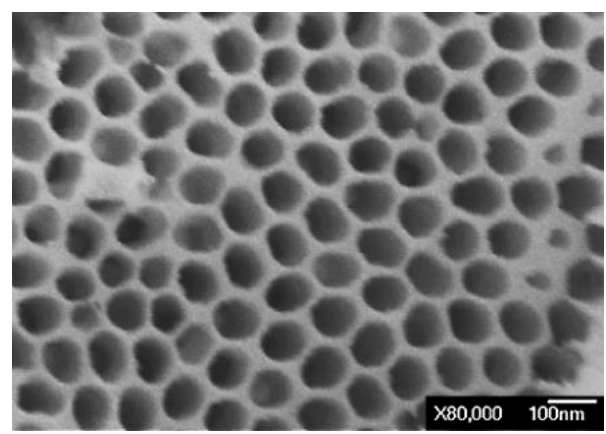

Figure 2. SEM image of alumina template with pores of size $80 \mathrm{~nm}$.

The nanopores in the AAO fabricated in the experiment are highly regularly arrayed. The diameter of the pores is $80 \mathrm{~nm}$, and the thickness of the tube wall increases with time. The template helps in fabricating nanowires, supporting good regularity. The AAO template and zinc film were placed in the die cast mold, as presented in Figure 3. The mold was depressurized to a vacuum of $10^{-3}$ torr, and heated at $750{ }^{\circ} \mathrm{C}$ for $60 \mathrm{~min}$. Then, a hydraulic force $\left(\mathrm{kgf} / \mathrm{cm}^{2}\right)$ was applied to cast the molten zinc into the nanopores of the AAO template. A period of condensation yields zinc nanowires.

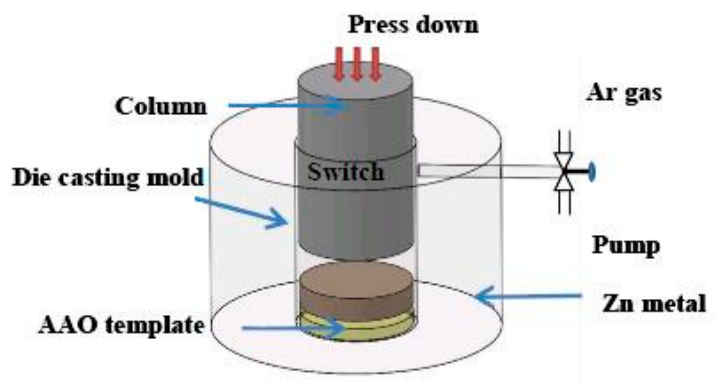

Figure 3. Die casting mold.

Let the applied pressure of the liquid metal that enters the AAO template be proportional to the surface tension of the liquid metal. The surface tension of zinc in the liquid state at $600{ }^{\circ} \mathrm{C}$ is 787 dyne/cm [16]. A hydraulic force was applied to overcome the surface tension of the liquid; the pressure of the liquid that enters a nanotube was calculated using Equation (1), where F denotes the normal force; $\mathrm{A}$ is the surface area of the sample; $\gamma$ is the surface tension of the liquid; $\theta$ is the contact angle between the liquid and the solid, and $\mathrm{r}$ is the diameter of the microtubules. As measured by a contact angle analyzer, the $\theta$ of AAO was $104.85^{\circ}$. The diameter of the AAO tube was $80 \mathrm{~nm}$. The surface area of the sample was $3.14 \mathrm{~cm}^{2}$. Therefore, the additionally applied force of zinc liquid entering the critical side of AAO was $3.16 \times 10^{8}$ dyne. 


$$
\mathrm{P}=\mathrm{F} / \mathrm{A}=-2 \gamma(\cos \theta / \mathrm{r})
$$

The zinc nanowires, following die casting, were placed in an atmospheric heat treatment furnace. The temperature of the furnace was increased to $300^{\circ} \mathrm{C}$ and maintained for $36 \mathrm{~h}$ to carry out oxidization to completely transform the zinc into zinc oxide nanowires [17]. A part of the AAO template was removed using $0.1 \mathrm{M}$ sodium hydroxide $(\mathrm{NaOH})$ solution to expose the nanowires. Since part of the template remains, the nanowires maintain their verticality, and the overall structure of the nanowires array has enough strength to facilitate the subsequent measurement of its piezoelectric properties. Finally, deionized water was used to perform ultrasonic cleaning to remove the residues after the reaction of the $\mathrm{AAO}$ template with $\mathrm{NaOH}$. Bake drying was performed on a heating platform. This concludes the fabrication of the $\mathrm{ZnO}$ nanowire piezoelectric component.

This paper uses SEM to observe the thickness of the AAO template, and an X-ray diffractometer (XRD) to analyze the crystal structure of nanowires. This study verifies that if nanowires are completely transformed into $\mathrm{ZnO}$ nanowires, then the crystal direction of $\mathrm{ZnO}$ nanowires of three lengths can be observed. Finally, a conductive atomic force microscope (C-AFM) is used to scan the nanowires in contact mode and measure the piezoelectric current. The length, width, and height of the AFM cantilever are $160 \mu \mathrm{m}, 50 \mu \mathrm{m}$, and $4.6 \mu \mathrm{m}$, respectively. The height and radius of the probe are $14 \mu \mathrm{m}$ and $7 \mathrm{~nm}$, respectively.

\section{Results and Discussion}

The surface morphology of nanowires is observed by scanning electron microscopy (SEM). Figure 4 shows the SEM images of a nanowire that is cast into an AAO template.

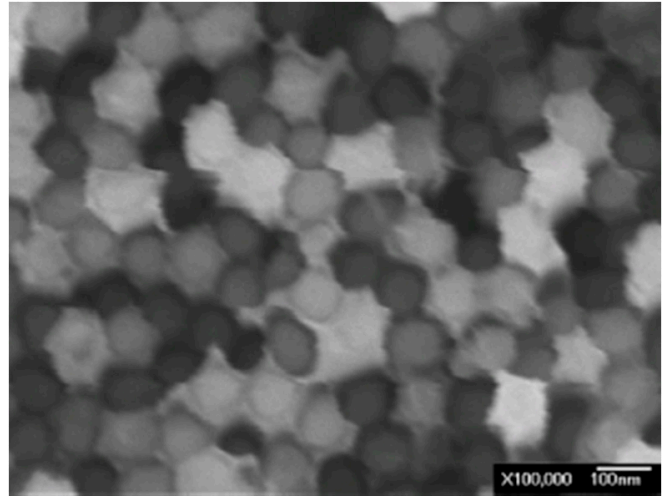

(a)

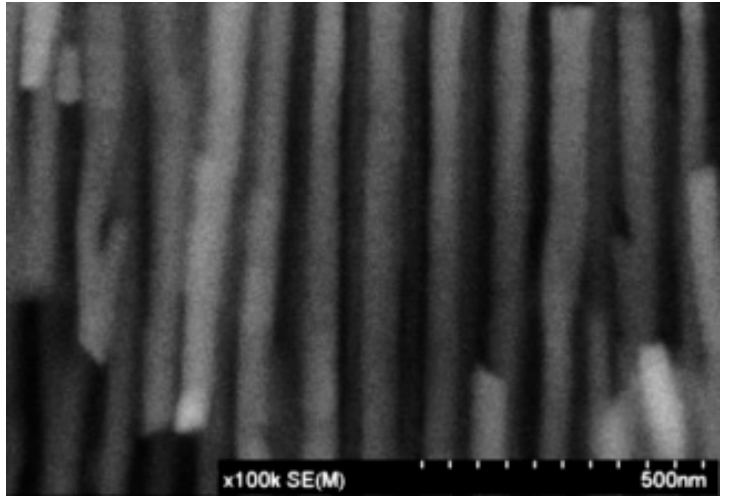

(b)

Figure 4. SEM images of nanowires that are cast into an AAO template: (a) top view; (b) lateral view.

Figure 5a shows the SEM front image of the partly removed AAO template. Figure $5 \mathrm{~b}$ displays the SEM image of the sample that is slanted $30^{\circ}$. In the figures, the well distribution of $\mathrm{ZnO}$ nanowires array can be observed. The $\mathrm{ZnO}$ nanowires are found to be very densely arrayed, and each exhibits good verticality. The SEM images in Figure 5a,b reveal that the diameter of the prepared $\mathrm{ZnO}$ nanowires is $80 \mathrm{~nm}$ and these nanowires are primarily well aligned on the bottom of the AOO template. Figure $5 \mathrm{c}$ presents the energy dispersive spectrometer (EDS) analytic chart. The area of EDS analysis is indicated by the blue square in Figure 5a. Table 1 presents the constituent elements of the fabricated nanowires. The table reveals that atmospheric annealing can transform zinc into $\mathrm{ZnO}$ nanowires. Additionally, to elucidate the crystalline quality of the prepared $\mathrm{ZnO}$ nanowires, Figure 6 presents a TEM image thereof. The prepared $\mathrm{ZnO}$ nanowires are single-crystal $\mathrm{ZnO}$ with a wurtzite structure and a $<0001>$ growth direction. 


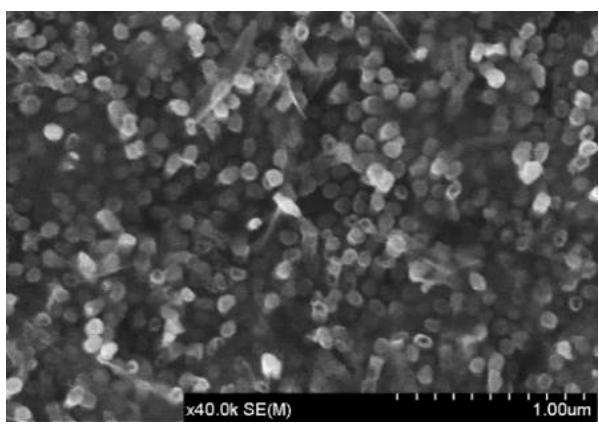

(a)

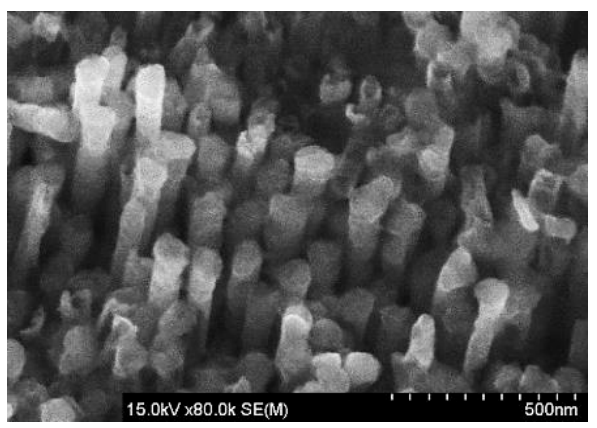

(b)

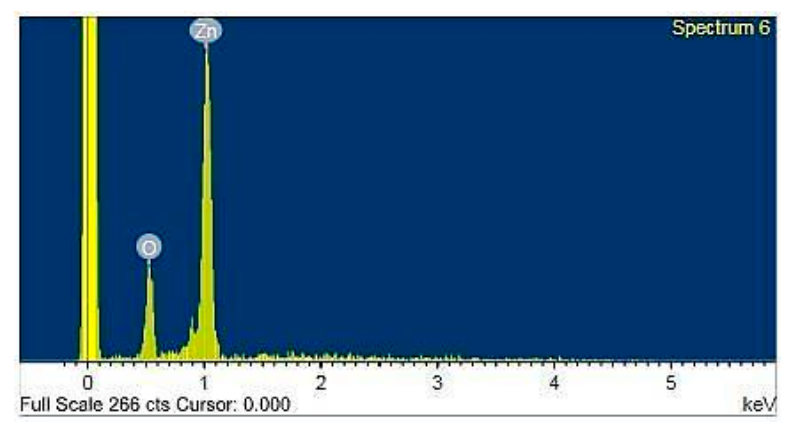

(c)

Figure 5. SEM images of $\mathrm{ZnO}$ nanowires: (a) top view; (b) lateral view, and (c) EDS pattern.

Table 1. EDS analytic composition of $\mathrm{ZnO}$ nanowire.

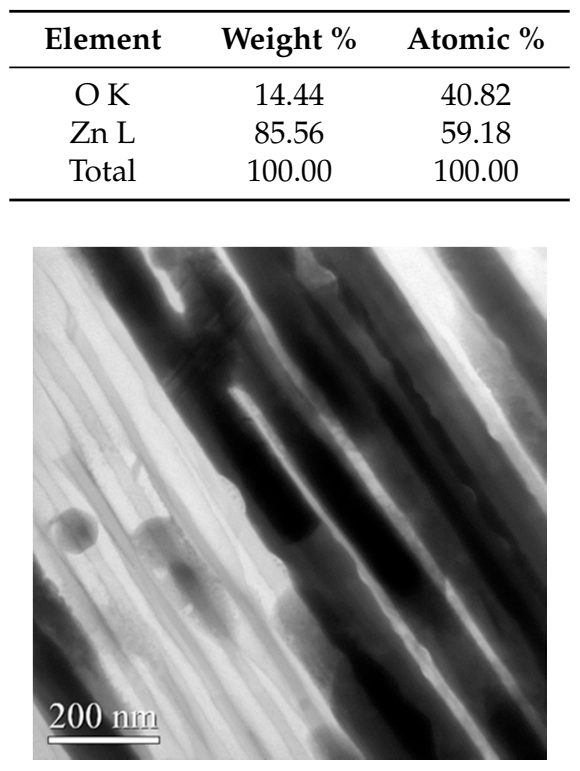

Figure 6. TEM image of prepared $\mathrm{ZnO}$ nanowires.

As shown in Figure 7, the AAO templates that were anodized for 5-8 h had thicknesses of $49.4 \mu \mathrm{m}$, $59.5 \mu \mathrm{m}, 68.1 \mu \mathrm{m}$, and $72.6 \mu \mathrm{m}$. The thickness of each template was determined by the duration of the second anodization. Figure 8 plots the relationship between the thickness of the template and the anodizing duration. When the anodizing time was $5-7 \mathrm{~h}$, the thickness increased at a rate of 9-10 $\mu \mathrm{m} / \mathrm{h}$, and when the anodizing time was $7-8 \mathrm{~h}$, the thickness increased at a rate of $4-5 \mu \mathrm{m} / \mathrm{h}$, because when the thickness of the template reached a certain value, the electrolyte could easily reach the bottom of the pores, so the growth rate was reduced. 


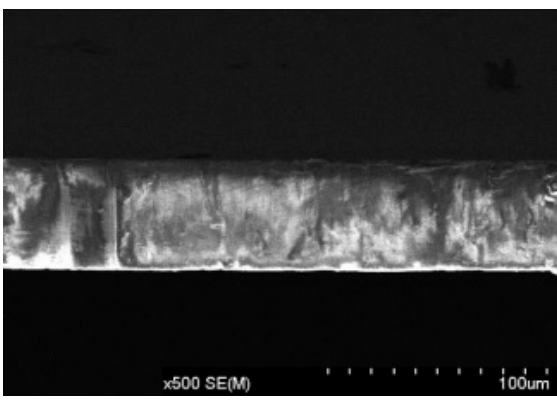

(a)

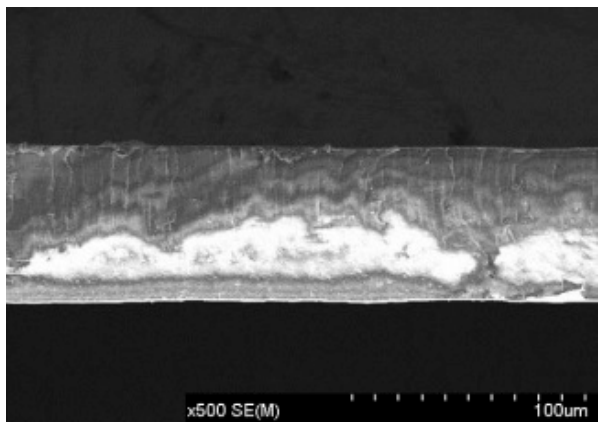

(c)

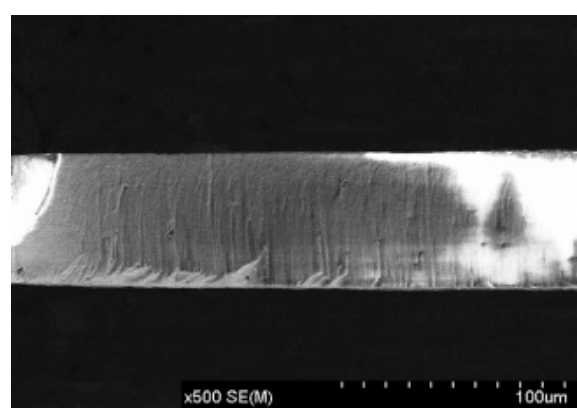

(b)

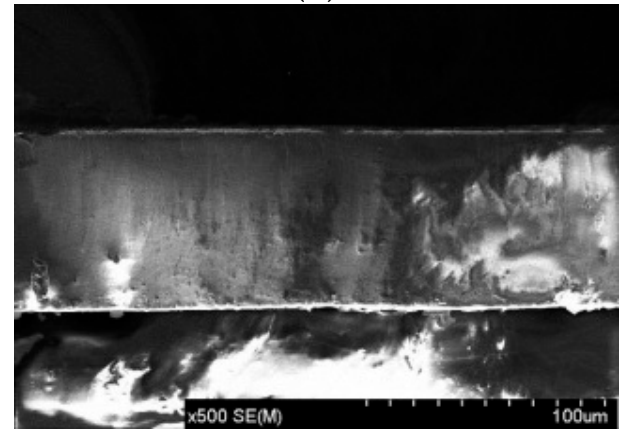

(d)

Figure 7. SEM images of the cross-section of alumina templates that were anodized for 5-8 h: (a) $5 \mathrm{~h}$, (b) $6 \mathrm{~h},(\mathbf{c}) 7 \mathrm{~h}$, and (d) $8 \mathrm{~h}$.

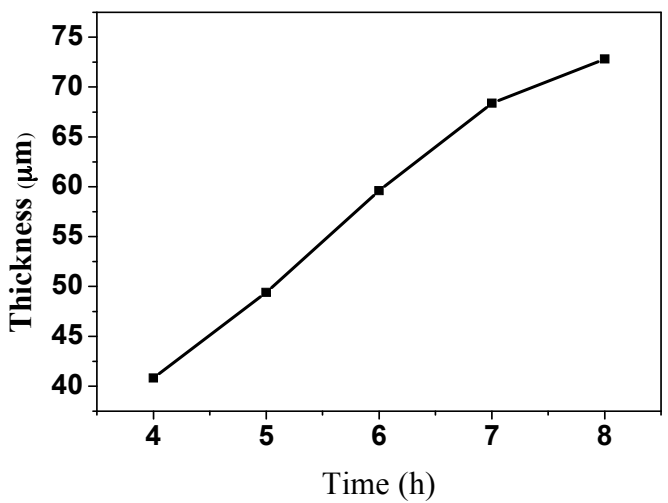

Figure 8. Relationship between thickness of alumina template and anodizing duration.

Figure 9 presents $\mathrm{XRD}$ diagrams that were obtained at $0.02^{\circ}$ intervals of diffraction angle $2 \theta$ from $30^{\circ}$ to $70^{\circ}$, following the XRD measurement of the $\mathrm{ZnO}$ nanowires that were made using the $\mathrm{AAO}$ template that was anodized for $5-7 \mathrm{~h}$. When the diffraction angle $2 \theta$ was approximately $34.3^{\circ}$, the $\mathrm{ZnO}$ nanowires of all three lengths yielded a very strong diffraction peak. Analysis of of the XRD chart and a comparison with JCPDS database revealed that the peak value was generated by the $\mathrm{ZnO}$ of the (002) side. Therefore, the $\mathrm{ZnO}$ nanowires that were fabricated in this work exhibit preferentially c-axis-oriented growth, and the peak strength tends to be proportional to the length of the nanowire. The chart also reveals that $\mathrm{Zn}$ nanowires have undergone heat treatment at a fixed temperature of $300{ }^{\circ} \mathrm{C}$ for $36 \mathrm{~h}$ are completely oxidized and thus transformed into $\mathrm{ZnO}$ nanowires.

$\mathrm{ZnO}$ exhibits non-ferroelectric piezoelectricity. Therefore, it cannot generate piezoelectricity by polarization, and only a structure that grows along the c-axis is piezoelectric. Therefore, preferentially c-axis-oriented $\mathrm{ZnO}$ is just highly suitable for fabricating piezoelectric components. 


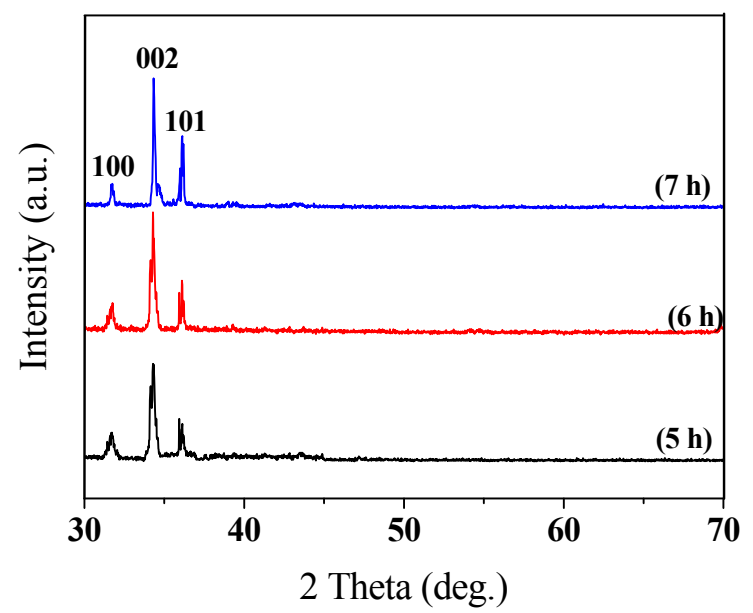

Figure 9. $\mathrm{XRD}$ patterns of $\mathrm{ZnO}$ nanowires that were fabricated using alumina template that was anodized for $5-7 \mathrm{~h}$.

AFM in contact mode was used to measure the surface morphology over $5 \mu \mathrm{m} \times 5 \mu \mathrm{m}$ areas of the surfaces of nanowires that were fabricated by the above experimental method. As shown in Figure 10, the surface morphology of the $\mathrm{ZnO}$ nanowires is very densely arrayed, and each $\mathrm{ZnO}$ nanowire exhibits good verticality.

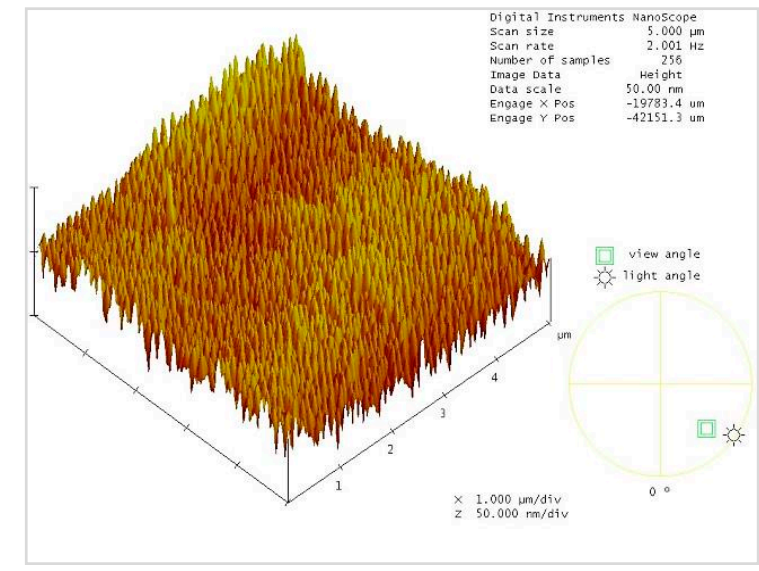

Figure 10. 3D diagram of surface morphology of $\mathrm{ZnO}$ nanowires, obtained using atomic force microscopy (AFM).

Conductive AFM was used to measure the piezoelectric currents of the $\mathrm{ZnO}$ nanowires of three lengths. Through the probe, stress was applied to deform the nanowires. The piezoelectric current that was generated on a certain area of each nanowire was measured. When a current was generated on a surface, the probe received a signal. An image of the current distribution on the scanned area was obtained. The current/voltage curve ( $I-V$ curve) at a particular point was obtained. Figure 11a-c plot the piezoelectric currents on $\mathrm{ZnO}$ nanowires that were fabricated using the alumina template that had been anodized for 5-7 h, obtained using C-AFM. As shown in the figures, the piezoelectric current increased with the length of the nanowire. The nanowire with a length of approximately $70 \mu \mathrm{m}$ that was fabricated after anodization for $7 \mathrm{~h}$ generates a current of $69 \mathrm{pA}$. Nanowires cause a deflection of a probe that is applied to stress them under scanning process. As the length of the nanowires increases, the probability of a deflection increases, and larger piezoelectric currents are measured. 


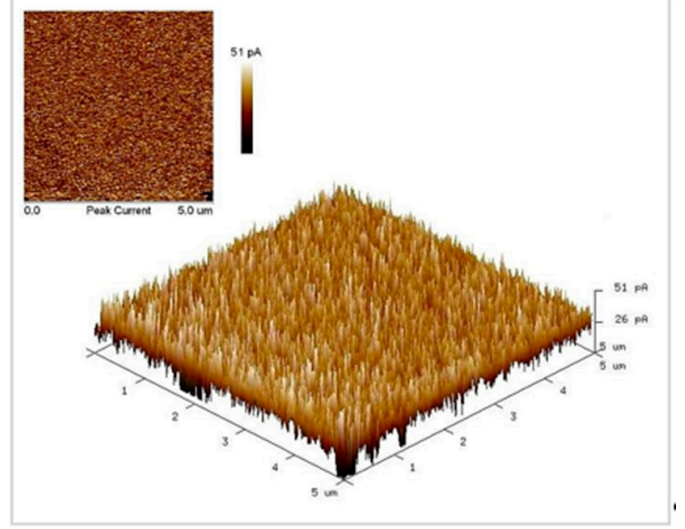

(a)

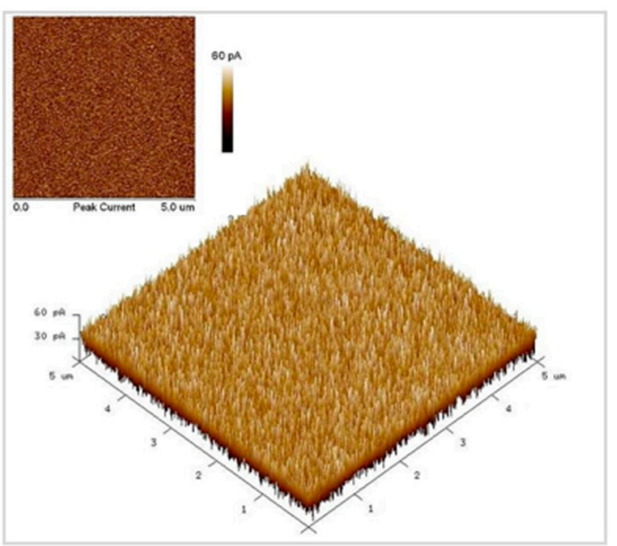

(b)

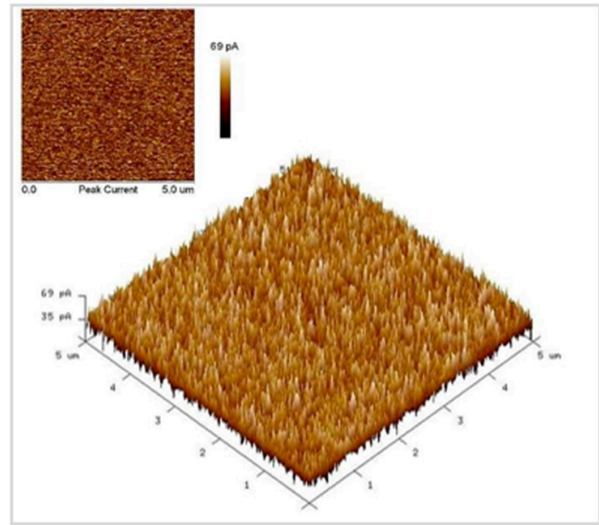

(c)

Figure 11. Piezoelectric current diagrams of $\mathrm{ZnO}$ nanowires, fabricated using alumina template that was anodized for $5-7 \mathrm{~h}$, measured using conductive atomic force microscopy (C-AFM): (a) $5 \mathrm{~h}$, (b) $6 \mathrm{~h}$, and $(\mathbf{c}) 7 \mathrm{~h}$.

Figure 12 plots the current/voltage property curve of $\mathrm{ZnO}$ nanowires measured using a platinum-plated probe serving as a metal electrode. Since Schottky contact exists between $\mathrm{ZnO}$ nanowires and metal electrodes, the current/voltage curve of $\mathrm{ZnO}$ nanowires has an asymmetric property and exhibits the property of a diode. Therefore, a piezoelectric component that is fabricated from $\mathrm{ZnO}$ generates a direct current.

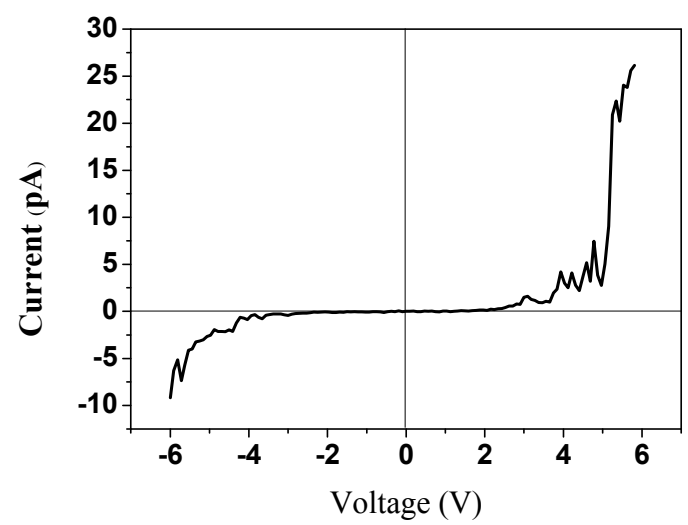

Figure 12. Current/voltage properties of $\mathrm{ZnO}$ nanowires, measured using a platinum-plated probe that serves as a metal electrode. 


\section{Conclusions}

In this work, AAO templates were made, the formation of nanopores was controlled, and a high-vacuum die casting technique was used to cast zinc into the nanopores of AAO. Zinc was transformed into $\mathrm{ZnO}$ nanowires by atmospheric heat treatment, and the AAO template was then removed to expose the nanowires. Microstructural analysis was carried out and observations made. Finally, the piezoelectric current that was generated by $\mathrm{ZnO}$ nanowires was measured using C-AFM. The results of this work are summarized as follows:

1. An aluminum template with a purity of $99.7 \%$ was anodized to produce an AAO template. The nanopores in the template were highly regularly arrayed and had a high aspect ratio. Finally, process parameters were optimized to minimize the cost of the required consumable materials.

2. The stress associated with the capillary phenomenon in die casting was calculated to obtain the normal force that was required to cast molten zinc metal into nanopores. The pressure was adjusted by using the controller of the die casting machine. A hydraulic force was used to cast molten zinc into the AAO template. After atmospheric heat treatment, arrayed $\mathrm{ZnO}$ nanowires were obtained.

3. The nanowires that were fabricated using the AAO template were very dense, had an aspect ratio of over 600 , were well arrayed, and exhibited good verticality.

4. The arrayed $\mathrm{ZnO}$ nanowires that were fabricated herein exhibited preferentially c-axis-oriented growth. The (002) peak strength was proportional to the length of the nanowire.

5. As observed from the test of piezoelectric properties, elucidated using C-AFM, longer nanowires generated a greater measured piezoelectric current. Among these $\mathrm{ZnO}$ nanowires, those fabricated using the alumina template that had been anodized for $7 \mathrm{~h}$ generated the greatest piezoelectric current of $69 \mathrm{pA}$.

Acknowledgments: The authors would like to thank the Ministry of Science and Technology of the Republic of China, Taiwan, for financially supporting this research under Contract No. NSC 102-2221-E-027-012.

Author Contributions: Chin-Guo Kuo is a consultant to the research and supervised the entire research work. Ho Chang is a main writer of the manuscript and advised the experiments. Jian-Hao Wang performed the experiments and assisted the research.

Conflicts of Interest: The authors declare no conflict of interest.

\section{References}

1. Wang, R.; King, L.H.; Sleight, W. Highly conducting transparent thin films based on zinc oxide. J. Mater. Res. 1996, 11, 1659-1664. [CrossRef]

2. Gao, Y.F.; Wang, Z.L. Electrostatic potential in a bent piezoelectric nanowire. The fundamental theory of nanogenerator and nanopiezotronics. Nano Lett. 2007, 7, 2499-2505. [CrossRef] [PubMed]

3. Gao, P.G.; Song, J.H.; Lin, J.; Wang, Z.L. Nanowire piezoelectric nanogenerators on plastic substrates as flexible power sources for nanodevices. Adv. Mater. 2007, 19, 67-72. [CrossRef]

4. Wang, Z.L.; Song, J. Piezoelectric nanogenerators based on zinc oxide nanowire arrays. Science 2006, 312, 242-246. [CrossRef] [PubMed]

5. Xue, X.; Nie, Y.; He, B.; Xing, L.; Zhang, Y.; Wang, Z.H. Surface free-carrier screening effect on the output of a $\mathrm{ZnO}$ nanowire nanogenerator and its potential as a self-powered active gas sensor. Nanotechnology $2013,24$. [CrossRef] [PubMed]

6. $\quad$ Lin, Y.; Deng, P.; Nie, Y.; Hu, Y.; Xing, L.; Zhang, Y.; Xue, X. Room-temperature self-powered ethanol sensing of a $\mathrm{Pd} / \mathrm{ZnO}$ nanoarray nanogenerator driven by human finger movement. Nanoscale 2014, 6, 4604-4610. [CrossRef] [PubMed]

7. Fu, Y.; Nie, Y.; Zhao, Y.; Wang, P.; Xing, L.; Zhang, Y.; Xue, X. Detecting liquefied petroleum gas (LPG) at room temperature using $\mathrm{ZnSnO} / \mathrm{ZnO}$ nanowire piezo-nanogenerator as self-powered gas sensor. ACS Appl. Mater. Interfaces 2015, 7, 10482-10490. [CrossRef] [PubMed]

8. Zeng, H.; Cao, Y.; Xie, S.; Yang, J.; Tang, Z.; Wang, X.; Sun, L. Synthesis, optical and electrochemical properties of $\mathrm{ZnO}$ nanowires/graphene oxide heterostructures. Nanoscale Res. Lett. 2013, 8. [CrossRef] [PubMed] 
9. Lin, S.; Hu, H.; Zheng, W.; Qu, Y.; Lai, F. Growth and optical properties of ZnO nanorod arrays on Al-doped $\mathrm{ZnO}$ transparent conductive film. Nanoscale Res. Lett. 2013, 8. [CrossRef] [PubMed]

10. Tian, X.; Pei, F.; Fei, J.; Yang, C.; Luo, H.; Luo, D.; Pi, Z. Synthesis and growth mechanism: A novel comb-like ZnO nanostructure. Phys. E 2006, 31, 213-217. [CrossRef]

11. Zhang, Z.; Ying, J.Y.; Dresselhaus, M.S. Bismuth quantum-wire arrays fabricated by a vacuum melting and pressure injection process. J. Mater. Res. 1998, 13, 1745-1748. [CrossRef]

12. Zhang, Z.; Gekhtman, D.; Dresselhaus, M.S.; Ying, J.Y. Processing and characterization of single-crystalline ultrafine bismuth nanowires. Chem. Mater. 1999, 11, 1659-1665. [CrossRef]

13. Chen, C.C.; Bisrat, Y.; Luo, Z.P.; Schaak, R.E.; Chao, R.E.; Lagoudas, D.C. Fabrication of single-crystal tin nanowires by hydraulic pressure injection. Nanotechnology 2006, 17, 367-374. [CrossRef]

14. Li, A.P.; Müller, F.; Birner, A.; Nielsch, K.; Gösele, U. Hexagonal pore arrays with a 50-420 nm interpore distance formed by self-organization in anodic alumina. J. Appl. Phys. 1998, 84, 6023-6026. [CrossRef]

15. Masuda, H.; Fukuda, K. Ordered metal nanohole arrays made by a two-step replication of honeycomb structures of anodic alumina. Science 1995, 268, 1466-1468. [CrossRef] [PubMed]

16. Hognes, T.R. The surface tensions and densities of liquid mercury, cadmium, zinc, lead, tin and bismuth. J. Am. Chem. Soc. 1921, 43, 1621-1628. [CrossRef]

17. Li, Y.; Meng, G.W.; Zhang, L.D. Ordered semiconductor ZnO nanowire arrays and their photoluminescence properties. Appl. Phys. Lett. 2000, 76, 2011-2013. [CrossRef]

(C) 2016 by the authors; licensee MDPI, Basel, Switzerland. This article is an open access article distributed under the terms and conditions of the Creative Commons by Attribution (CC-BY) license (http://creativecommons.org/licenses/by/4.0/). 